\title{
Implementation of Asynchronous Predictive Fetch to Improve the Performance of Ajax-Enabled Web Applications
}

\author{
Andi Ahmad Dahlan \\ College of Information Science and Engineering \\ Ritsumeikan University \\ 1-1-1 Nojihigashi, Kusatsu City, \\ Shiga Prefecture, Japan 525-8577 \\ $+81-77-561-4971$ \\ aadfuty@gin.ics.ritsumei.ac.jp
}

\author{
Toshikazu Nishimura \\ College of Information Science and Engineering \\ Ritsumeikan University \\ 1-1-1 Nojihigashi, Kusatsu City, \\ Shiga Prefecture, Japan 525-8577 \\ $+81-77-561-4971$ \\ tnt@is.ritsumei.ac.jp
}

\begin{abstract}
An implementation study of Asynchronous Predictive Fetch method to improve the performance of Ajax-enabled Web applications is presented. To evaluate the performance of the approach, two versions of Ajax-enabled Web applications that implemented the same user interface were developed. A number of trials were accomplished to collect performance data on each application when doing the same tasks. We found that the method provided a performance improvement of the average response time of $64.5 \%$.
\end{abstract}

\section{Categories and Subject Descriptors}

H.5.3 [Information Interfaces and Presentation]: Group and Organization Interfaces - web-based interaction, asynchronous interaction.

\section{General Terms}

Performance, Experimentation, Measurement.

\section{Keywords}

Ajax, Predictive Fetch, Web Application, Performance.

\section{INTRODUCTION}

Ajax, a term first coined by Jesse James Garret [7], is really a set of technologies that provide a new way of designing and developing Web applications. The technologies work together in different parts of the application, each of which provides specific functionality to collectively create a powerful Web application [9]. Google Maps, Google Suggest, Yahoo Mail, Gmail and A9 Product Search are several examples of the real world

Permission to make digital or hard copies of all or part of this work for personal or classroom use is granted without fee provided that copies are not made or distributed for profit or commercial advantage and that copies bear this notice and the full citation on the first page. To copy otherwise, or republish, to post on servers or to redistribute to lists, requires prior specific permission and/or a fee.

iiWAS2008, November 24-28, 2008, Linz, Austria.

(c) 2008 ACM 978-1-60558-349-5/08/0011 \$5.00. applications that show the usefulness of this technique.

One of the primary goals of Ajax implementation on Web applications is to improve the user's experience of response time. The response time is defined as the time between sending a request and receiving the response. By reducing response time, Ajax can provide a significantly better user experience. Ajax can improve response time by communicating with the server without full-page requests. In addition, the response time of Ajax Web applications can be further improved by implementing a Web prefetching technique such as Asynchronous Predictive Fetch used in this paper.

Web prefetching is a technique that is commonly applied to reduce the access latency perceived by the Web user. This technique enables a Web application to prefetch data from the server so that it is immediately available upon user actions. It is clear that the Web application must prefetch using accurate information in order to achieve reasonable performance that justifies the additional resources consumed (bandwidth, extra server load) [2].

\section{MOTIVATION}

Even though the implementation of Ajax in Web applications is gaining popularity, few researches have been published showing the performance of the Ajax applications compared to the traditional Web applications in which full-page refresh is always implemented. Merill's application test[4] found the total bandwidth savings of $61 \%$ due to the use of Ajax approach. White [1] reported that there was $73 \%$ improvement in the number of bytes transferred in the Ajax application over the traditional version. He also showed that the user could save on average $32 \%$ of the time required to complete the tasks in the Ajax version. However, the two applications did not implement the same user interface and the impact of sales personnel's skill that could significantly affect the reported figure was not investigated. C. Smullen and S. Smullen $[5,6]$ found that a typical user would see a performance increase in the response size of $55 \%$ and a mean service time improvement of approximately $16 \%$. 
All these works show that Ajax applications perform better than classic Web applications in terms of response size and service time. However, their investigations were mainly focused on reducing the bandwidth use through the implementation of Ajax. This proposed work tries to improve the performance of Ajaxenabled Web applications in terms of user's experience of response time by implementing Asynchronous Predictive Fetch approach.

\section{ASYNCHRONOUS PREDICTIVE FETCH}

Asynchronous Predictive Fetch is a kind of Web prefetching techniques that implements both the prediction and the prefetching engine in the client's Web browser, programmed in JavaScript language code. We refer to this method as Predictive Fetch in references $[10,11]$.

To help predict what the user will do next, the Asynchronous Predictive Fetch could potentially use some hints such as a user's profile and current activity. In our implementation case, however, we use cues derive from a user's current actions through the user interface. In this context, the user's current actions are divided into two categories:

- Synchronous user actions are the actions done by a user, such as clicking a link or a button, resulting in a new response displayed on the screen.

- Asynchronous user actions, on the other hand, are the actions done by a user, such as hovering a mouse pointer over a button or selecting an item in a dropdown list, causing no change in the currently displayed page on the screen.

Based on the above definitions, Asynchronous Predictive Fetch approach in this research is employed by utilizing information derived from asynchronous user actions.

The prefetching engine employed in the Asynchronous Predictive Fetch is an Ajax engine. It is the engine that relies on the XMLHttpRequest object responsible for making requests to, and handling responses from the server. The Ajax engine may have more than one XMLHttpRequest object to make multiple requests simultaneously.

Asynchronous Predictive Fetch requires a cache in order to keep all prefetched responses. In our implementation case, we use the Browser-side Cache [10] that is a JavaScript object that holds the request and its corresponding response in pairs. The requests are the cache keys and the responses are the cache values. When the browser is to make a request the server, the cache will be first interrogated. If the request is in the cache, it means that the corresponding response must have already been in there. All the application scripts need to do is just pick the response up and display it to the user's screen. Further request processing to the server is then unnecessary.

\section{IMPLEMENTATION}

The application used in our implementation case is a sample Web application that provides academic-related information to students in our campus, Polytechnic, Andalas University,
Indonesia. Information that are extracted either statically from files or dynamically from a database include information of campus' academic year, syllabus or course information for each study program, students' personal data and transcript of academic records. The application is built based on MVC (Model-View-Controller) design pattern. The Web server is Apache installed on Windows Operating System. The application uses PHP 5, Smarty template engine and PostgreSQL database server. All responses retrieved from the server are coded in XHTML format.

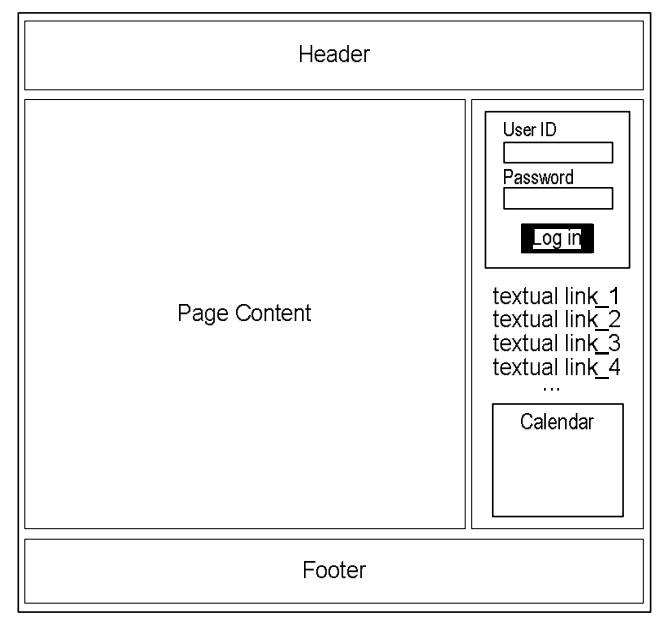

Figure 1. User interface layout

Figure 1 shows the user interface layout of our sample Web application. The header and footer parts are static views. The page contents on the left side, however, are dynamically updated to display the information requested by the user. On the right side of the layout locates a $\log$ in form, publicly accessible links, and a calendar box. Some user-specific links, e.g. additional links for students, will be added to the current links when he or she successfully logs in the system. Keyboard input such as the Tab and the Enter buttons, and a mouse pointer can be used to navigate over the links and form elements.

When the user moves the mouse pointer over a textual link, the mouseover event on that link is fired. If the event handler capturing this event is designed to invoke an Asynchronous Predictive Fetch process, it will then ask the XMLHttpRequest for making a request to the server. This request corresponds to the link on which the mouseover event is triggered. While the request is in progress, the user may quickly move the mouse pointer over another link without clicking the previous link. Again, another request may be accomplished if the event handler specific to the link is also designed to do the same thing as before while the user does not actually click the link. As a consequence, these two user actions will make two unwanted requests resulting wasted bandwidth.

To reduce the impact on user actions as described above, the prefetching mechanism of Asynchronous Predictive Fetch should not solely rely on the onmouseover event to a link in order to start making an asynchronous request. Instead, we combine the onmouseover event with an estimated delay time before finally calling the server for a request. By this approach, when the user 
moves the mouse pointer over a link, a timer will be set. If the user quickly moves his mouse out of the boundary of current link while the timer is not fired yet, the onmouseout event triggered by the user action will reset the previous timer function on that link causing no prefetching process will be taken. In the other way, if the user is still hovering the mouse pointer over that link and the timer is eventually fired, then an asynchronous call will be accomplished. However, determining the value of delay time is not an easy work. If we set the time too short, say 50 milliseconds, excessively un-demanded requests may result. On the other hand, if the delay time is too long, say 10 seconds, the prefetching function may not work at all.

To help estimating an appropriate delay time, a heuristic data related to various speeds of a user's actions through the user interface should be collected before developing an application. After having done some experiments, we define several heuristics as follows:

- When a user hovers/moves a mouse over a textual link and clicks it, how long the time duration is needed to do the click action? (onmouseover $\longrightarrow$ onclick)

- A very fast action takes around $125-250$ milliseconds.

- A fast action takes around $250-850$ milliseconds.

- A normal action takes more than 850 milliseconds.

- When a user hovers/moves a mouse over a button and clicks it, how long the time duration is needed to do the click action? (onmouseover $\longrightarrow$ onclick)

- A very fast action takes around $125-250$ milliseconds.

- A fast action takes around $250-750$ milliseconds.

- A normal action takes more than 750 milliseconds.

- When a user selects an item in a drop-down list and clicks on the 'Go' button, how long the time duration is needed? (onchange $\longrightarrow$ onclick)

- A very fast action takes around $350-450$ milliseconds.

- A fast action takes around $450-1000$ milliseconds.

- A normal action takes more than 1000 milliseconds.

The user's speed of using a mouse is relatively same as that of a keyboard. However, in many cases, using a keyboard button seems to take more time than using a mouse pointer, because it is relatively difficult to make sure that an element is getting focused before pressing the Enter button on the keyboard to start making a request.

\subsection{Asynchronous User Actions}

The implementation of Asynchronous Predictive Fetch in our Web application is developed by using a heuristic approach. The heuristic is based on specific contexts of a user's intentions and actions through the user interface. The user's intention is derived by investigating all kinds of information that are provided by the application. Some user's intentions may involve more than one user's action. In this case, the actions are divided into sub-goal actions and a main-goal action, the final action to realize the user's intention.

Some of the user's actions through the user interface of our Web application are described as follows:
- If a mouse pointer is hovered over a link for several milliseconds, say 250 milliseconds, anticipate the user's click action on that link by requesting the appropriate data in advance.

- If a user is willing to $\log$ in to the system by filling his/her user name and password in the log-in form, make an asynchronous call to the server as soon as the mouse pointer hovers over the submit button of the form.

- If a user is going to see a course list of a study program, make an asynchronous call to the server right after the user has just selected an item in a drop-down list anticipating the user's click action on the 'Go' button.

\subsection{Synchronous User Actions}

In the previous section, we define synchronous user actions as the actions done by a user, such as clicking a link or a button, resulting in a new response displayed on the screen. Instead of simply making a request and keeping the response in the cache as what the asynchronous user actions really do it, the synchronous user actions are the actions that are intentionally done by the user to view that something is updated in the user interface.

\subsection{Measurement Procedure}

Measuring the time to complete a task is one of the most useful metrics when looking at the success of an Ajax implementation. The actual measurement process consists of three steps: identifying a task's starting and ending points, adding procedures to measure the starting and ending times, and combining multiple data measurements to gain useful information [8].

In our implementation case, we are recording the time to access information of campus' academic year, syllabus or course information for each study program, students' personal data and transcript of academic records. These activities are done by the user through the user interface shown in the user's machine. All recording processes are then carried out in the user's machine by including recording scripts, written in JavaScript code, within the application. In addition to collecting the process-time data, our implementation case is also measuring the response size or bandwidth use for the entire process. The measurement process is also performed in the user side by using a measurement tool (http://www.webperformanceinc.com/analyzer/).

\section{DATA COLLECTION}

To measure the performance of proposed research, two versions of Ajax-enabled Web applications were developed. One version was developed by implementing synchronous Ajax only (labeled as AJAX hereafter), and the other version was built with the capability of Asynchronous Predictive Fetch (labeled as APFAJAX hereafter). Both the Ajax applications render the same "look and feel" user interface at the presentation level.

The metric used to evaluate the performance of the Ajax-enabled Web applications is mainly based on the user's experience of response time that is the application responsiveness. Nevertheless, investigating the bandwidth consumed for the 
entire process, by measuring the total size transferred during a test case session, is also taken into account.

For this to be a fair comparison, several guidelines were used in the execution of the test case:

- The test case consisted of three sessions marked as Session A, B and C, respectively. Each of which consisted of a fixed number of tasks and was accomplished by a user in the same order.

- Before conducting the test case, a measurement tool was launched in the user's machine.

- Before starting collecting data for one session, the server was "warmed up" by taking some readings of data [3]. This was done because it was never known how different parts of the application might affect initial performance, for example, if a SQL database was hit, the first query might cache the SQL results, so subsequent queries were faster.

- After warming up the server, the browser's built-in cache was cleared. This simulated hitting the server for the very first time.

- Session A, B, and C consisted of 20, 27, and 34 numbers of specific tasks or queries, respectively. Each of the test case session was conducted 20 times on several days through the Internet.

All the tests were done by the author performing various speeds of a user's actions through the user interface as described in the previous section.

\section{ANALYSIS}

Relying on the true power of Ajax that can asynchronously interact with the server is the main reason to have a big opportunity to improve the response time of performed user's actions. Asynchronous interaction means that we can ask the server for only small amount of data required to update the current user's display. It results in less time to process a request in the server, to send the response to the client machine, and to render it on the user interface. Furthermore, having the ability to prefetch data in advance in anticipating the user's actions can make the effort of decreasing the response time much more achievable.

Incorporating Asynchronous Predictive Fetch in an Ajax Web application would result in better application responsiveness. However, in some cases, this might not be true. When the user starts making a request by clicking a link for instance, our implementation algorithm may get informed that an asynchronous predictive fetch (APF) process for that request has just been launched. It then forces the synchronous user action (SUA) process idle for a moment while waiting the APF process to complete its job. Unfortunately, for something unknown happens in the network resulting in a bit long downloading time or since the response size is too big, the timeout delay set in the SUA process is over while the APF process is still accomplishing its work. At that time, the SUA process then begin making its own request to the server and it finally gets the response and renders it to the user interface. What can be learned from this possible case is that the APF-AJAX implementation may introduce worst application responsiveness than does the AJAX application. This is because there is an extra waiting time needed before the SUA process finally gets started.

We could hardly cope with the first cause explained the above scenario, because the problem has likely nothing to do with the server and the Web application running on it. The second cause, however, could be addressed by applying some strategies. One approach is that we may perform such a profiling test to measure the typical response time needed to download the typical document size of our resources that would serve the user. It is not easy to figure out the size and its corresponding download time to all bits and pieces of data we have. However, in our implementation case, we found that estimating the typical size of student's personal data or syllabus, for instance, was not too difficult. All students relatively had the same amount of data size. Most of data size we had in our database was ranging from around $5 \mathrm{~KB}$ to $50 \mathrm{~KB}$ in size, with around $10 \mathrm{~KB}$ in the average. So we tried to measure the response time of those estimated data sizes through the Internet connection. By using our measurement tool, we also enabled to observe other websites in the Internet and helped us to draw such a baseline data. After having the approximation values of the response time for specific data size, we then got an idea to estimate the timeout delay needed for specific user requests. In our implementation case the timeout delay is around $500-1,000 \mathrm{msec}$.

Another method that can be used to address the aforementioned issue is start pushing the response to the screen, without waiting until the whole response successfully downloaded, by using the JavaScript setInterval function that would run every few milliseconds. This can be realized by capturing the current readyState value of the APF process making the corresponding request. This strategy can improve the perceived performance, that is, the speed is vastly faster when in fact the total amount of download time is roughly similar, but it makes a dramatic perceived difference [3].

To calculate the performance improvement that can be achieved through the implementation of Asynchronous Predictive Fetch approach to an Ajax-enabled Web application, we define the Performance Improvement (PI) [1] as:

$$
\frac{(\text { AJAX value }- \text { APF-AJAX value) }}{\text { AJAX value }}
$$

The PI value denotes the percentage improvement of the APFAJAX application compared to the AJAX application developed in our implementation case. Any positive value states that there is a saving gained by using the APF-AJAX application. The larger the value is, the more the saving would be. Conversely, any negative value states the other, opposite condition.

Table 1 shows the summary of the average response times and their corresponding PI for each test case session. As can be seen in the Table 1 , the performance improvement related to the response time that is gained due to the use of APF-AJAX is over $62 \%$ on all the test case sessions. It means that we can save our 
time about $62 \%$ when accessing the APF-AJAX application compared to that of the AJAX application.

Table 1. Summary of PI by the average response time

\begin{tabular}{|c|c|c|c|}
\hline \multirow{2}{*}{$\begin{array}{c}\text { Application } \\
\text { Type }\end{array}$} & \multicolumn{3}{|c|}{ Average Response Time (msec.) } \\
\cline { 2 - 4 } & Session A & Session B & Session C \\
\hline AJAX & 7,571 & 9,675 & 13,475 \\
\hline APF-AJAX & 2,667 & 3,135 & 5,108 \\
\hline PI (\%) & 64.8 & 67.6 & 62.1 \\
\hline
\end{tabular}

The data comparison also reveals that the user's experience of response time is not affected by the number of tasks or queries the user did during accessing to information of the Web application. The number of tasks accomplished in the Session B during the test is fewer than that of in Session C. However, the former test case shows a better performance improvement, $67.6 \%$ over $62.1 \%$ of time savings. In contrast, the number of tasks performed in the Session B is more than that of in Session A. Again, the former shows a better performance improvement, $67.6 \%$ over $64.8 \%$ of time savings.

Table 2. Response time for all the test case sessions

\begin{tabular}{|c|c|c|c|c|}
\hline \multirow{2}{*}{$\begin{array}{c}\text { Application } \\
\text { Type }\end{array}$} & \multicolumn{4}{|c|}{ Response Time (msec.) } \\
\cline { 2 - 5 } & Min & Mean & Max & Total \\
\hline AJAX & 80 & 379 & 1,196 & 30,721 \\
\hline APF-AJAX & 10 & 135 & 980 & 10,910 \\
\hline PI (\%) & 87.6 & 64.5 & 18.1 & 64.5 \\
\hline
\end{tabular}

Table 2 presents summary of the minimum, mean, maximum, and total response time and their corresponding PI values accumulated from the whole test case sessions. Inspecting information presented in the Table 2, we may assume that the performance improvement of response time due to the use of APF-AJAX seems to be linear to the amount of time needed to download and display the response retrieved from the server. The lower response time is, the greater the performance increase will do. With a time of 80 milliseconds needed by the AJAX to download a response, the performance improvement reaches around $88 \%$, while downloading a response with the estimated download time of around one second, the performance improvement is only about $18 \%$. The average PI, however, is $64.5 \%$.

Figure 2 plots a visual graph of the relationship between response time perceived by the AJAX and the corresponding performance improvement that could be achieved by the implementation of APF-AJAX. It can be seen in Figure 2 that the performance increase is not completely linear to the amount of time needed by the browser to the download a response. Even though the download time of a response needed by AJAX is lower, say 134 milliseconds, the PI value is much lower compared to that of a $450-\mathrm{msec}$. response ( $43 \%$ vs $93 \%)$. It means that the performance increase of response time is improvable even the download time is long. This phenomenon can be learned by examining the graph curve on rightmost side of the Figure 2.

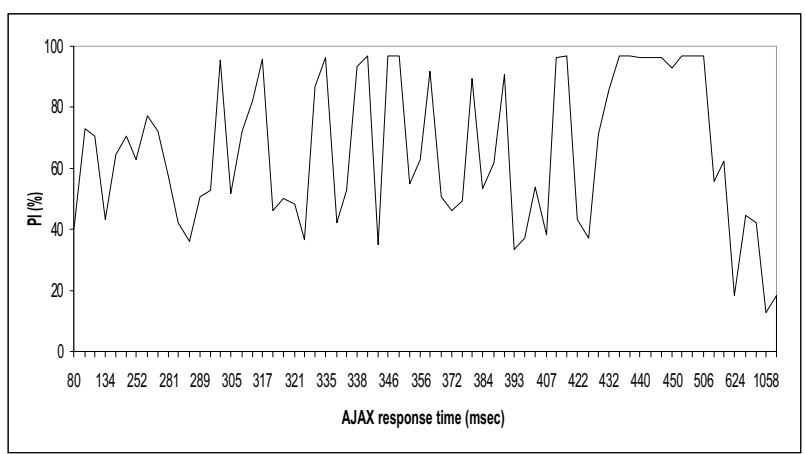

Figure 2. PI versus AJAX response time

Summary of the average values of total transferred data size, the bandwidth use, and their corresponding PI is shown in Table 3. The negative values of PI denote the performance decrease due to the use of APF-AJAX application. This performance decrease is resulted from two issues related to the APF-AJAX implementation. Firstly, the client's browser needs to download additional resources, the JavaScript code, to manage the functionality of Asynchronous Predictive Fetch process in the client side. Secondly, in most cases, the APF-AJAX application prefetches information from the server in anticipation of the user's actions. As a result, some amounts of resources that have been downloaded and kept in the Browser-side Cache are not used.

Table 3. Summary of PI by the total data transfer

\begin{tabular}{|c|c|c|c|}
\hline \multirow{2}{*}{$\begin{array}{c}\text { Application } \\
\text { Type }\end{array}$} & \multicolumn{3}{|c|}{ Total Size (KB) } \\
\cline { 2 - 4 } & Session A & Session B & Session C \\
\hline AJAX & 244 & 268 & 375 \\
\hline APF-AJAX & 270 & 280 & 403 \\
\hline PI (\%) & -10.8 & -4.5 & -7.6 \\
\hline
\end{tabular}

Another thing that can be learned from Table 3 is that the performance decrease has nothing to do with the number of tasks or queries performed during the test case sessions. Session $C$ takes more tasks or queries than Session B so the performance decrease introduced by Session $C$ is higher than does Session B $(-7.6 \%$ over $-4.5 \%)$. However, compared to Session A, the performance decrease resulted from Session $C$ is lower $(-7.6 \%$ against $-10.8 \%$ ).

Figure 3 draws the performance decrease that was perceived due to the implementation of APF-AJAX. It is shown in Figure 3 that in the worst case, the performance decrease introduced by APFAJAX application could reach $27 \%$ compared to AJAX. In most cases, however, the performance decrease is lower than $3 \%$. Furthermore, the deployment of broadband technologies is now commonplace, so the wasted bandwidth due the implementation of Asynchronous Predictive Fetch is not a big issue. 


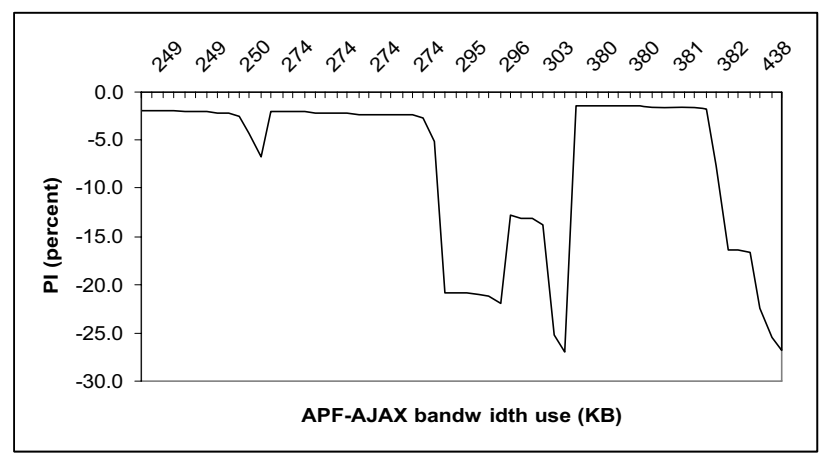

Figure 3. PI versus APF-AJAX bandwidth use

\section{CONCLUSION AND FUTURE WORK}

We have proposed the implementation of Asynchronous Predictive Fetch to improve the performance of Ajax-enabled Web applications. Through the implementation case, we found that this method provided a performance improvement of average response time of $64.5 \%$. So we can conclude that the users' experience of response time is improved significantly due to the implementation of the Asynchronous Predictive Fetch approach.

The performance improvement of user's experience of response time is not affected by the number of tasks or queries done by a user. It mainly depends on the user's behaviors through the user interface and the accuracy level of prediction method applied in the application. Related to these aspects, we also found that the performance improvement is not linear to the amount of time needed to download a response. However, by providing a smallsize response that requires less download time, the performance improvement of at least $33 \%$ can be likely achieved. Fortunately, the nature of Ajax that enables Web applications to partially refresh the user interface by transferring less data makes this "rule of thumb" achievable. Moreover, by the power of making as many as data requests behind the scene, the Asynchronous Predictive Fetch would be able to satisfy this rule.

In accordance with our implementation study, there are issues that would be further addressed. We wish to know how much the performance improvement of response time experienced by the user is affected by large data sizes downloaded from the server.
We are also looking forward to evaluating the effectiveness of the approach by combining with other prefetching hints such as the user's profile and history and information of the user's current activities on the user interface.

\section{REFERENCES}

[1] Alexei White, "Measuring the Benefits of Ajax", http://www.developer.com/xml/article.php/3554271.

[2] B. De La Ossa, et al., "Delfos: the Oracle to Predict Next Web User's Accesses", Proceedings of $21^{\text {st }}$ International Conference on Advanced Networking and Application, IEEE, 2007.

[3] Brad Neuberg, "Tutorial: How to Profile and Optimize Ajax Applications", http://codinginparadise.org/weblog/2006/08/ tutorial-how-to-profile-and-optimize.html, 2006.

[4] C. L Merill, "Using Ajax to Improve the Bandwidth Performance of Web Applications", Web Performance. Inc, 2006.

[5] C. Smullen and S. Smullen, "Modelling AJAX Application Performance", 524-074, Web Technologies, Applications, and Services 2006, ed. J.T. Yao, ACTA Press, Calgary, 2006.

[6] C. Smullen and S. Smullen, "Ajax Application Server Performance", Proceedings of the IEEE SoutheastCon 2007, Richmond, Virginia, pp. 154-158, March 22-25, 2007.

[7] Jesse James Garret, “Ajax: A New Approach to Web Applications", http://www.adaptivepath.com/ideas/essays/ archives/ 000385.php, 2005.

[8] Joshua Eichorn, "Understanding AJAX: Using JavaScript to Create Rich Internet Applications", Prentice Hall, USA, 2006.

[9] Matthew Eernisse, "Build Your Own AJAX Web Applications", SitePoint Pty. Ltd., USA, 2006.

[10] Michael Mahemoff, “Ajax Design Patterns”, O'Reilly Media, Inc., USA, 2006.

[11] Nicholas C. Zakas, et al., "Professional Ajax $2^{\text {nd }}$ Edition", Wiley Publishing, Inc., USA, 2007. 